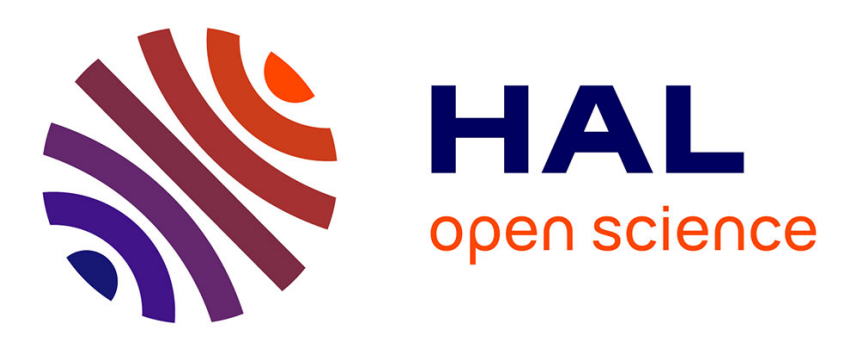

\title{
Maximal Decidable Fragments of Halpern and Shoham's Modal Logic of Intervals
}

Angelo Montanari, Gabriele Puppis, Pietro Sala

\section{To cite this version:}

Angelo Montanari, Gabriele Puppis, Pietro Sala. Maximal Decidable Fragments of Halpern and Shoham's Modal Logic of Intervals. Proceedings of ICALP 2010, 2010, Bordeaux, France. pp.345-356, 10.1007/978-3-642-14162-1_29. hal-00717788

\section{HAL Id: hal-00717788 \\ https://hal.science/hal-00717788}

Submitted on 30 Oct 2013

HAL is a multi-disciplinary open access archive for the deposit and dissemination of scientific research documents, whether they are published or not. The documents may come from teaching and research institutions in France or abroad, or from public or private research centers.
L'archive ouverte pluridisciplinaire HAL, est destinée au dépôt et à la diffusion de documents scientifiques de niveau recherche, publiés ou non, émanant des établissements d'enseignement et de recherche français ou étrangers, des laboratoires publics ou privés. 


\title{
Maximal decidable fragments of Halpern and Shoham's modal logic of intervals
}

\author{
Angelo Montanari ${ }^{1}$, Gabriele Puppis ${ }^{2}$, and Pietro Sala ${ }^{1}$ \\ 1 Department of Mathematics and Computer Science, Udine University, Italy \\ \{angelo.montanari, pietro.sala\}@dimi.uniud.it \\ 2 Computing Laboratory, Oxford University, England \\ gabriele.puppis@comlab.ox.ac.uk
}

\begin{abstract}
In this paper, we focus our attention on the fragment of Halpern and Shoham's modal logic of intervals (HS) that features four modal operators corresponding to the relations "meets", "met by", "begun by", and "begins" of Allen's interval algebra ( $A \bar{A} B \bar{B}$ logic). $A \bar{A} B \bar{B}$ properly extends interesting interval temporal logics recently investigated in the literature, such as the logic $B \bar{B}$ of Allen's "begun by/begins" relations and propositional neighborhood logic $A \bar{A}$, in its many variants (including metric ones). We prove that the satisfiability problem for $A \bar{A} B \bar{B}$, interpreted over finite linear orders, is decidable, but not primitive recursive (as a matter of fact, $A \bar{A} B \bar{B}$ turns out to be maximal with respect to decidability). Then, we show that it becomes undecidable when $A \bar{A} B \bar{B}$ is interpreted over classes of linear orders that contains at least one linear order with an infinitely ascending sequence, thus including the natural time flows $\mathbb{N}, \mathbb{Z}$, and $\mathbb{R}$.
\end{abstract}

\section{Introduction}

For a long time, the role of interval temporal logics in computer science has been controversial. On the one hand, it is commonly recognized that they provide a natural framework for representing and reasoning about temporal properties in many computer science areas (quoting Kamp and Reyle [11], "truth, as it pertains to language in the way we use it, relates sentences not to instants but to temporal intervals"), including specification and design of hardware components, concurrent real-time processes, event modeling, temporal aggregation in databases, temporal knowledge representation, systems for temporal planning and maintenance, qualitative reasoning, and natural language semantics [9]. On the other hand, the computational complexity of most interval temporal logics proposed in the literature has been a barrier to their systematic investigation and their extensive use in practical applications. This is the case with the modal logic of time intervals HS introduced by Halpern and Shoham in [10]. HS makes it possible to express all basic binary relations that may hold between any pair of intervals (the so-called Allen's relations [1]) by means of four unary modalities, namely, $\langle B\rangle,\langle E\rangle$ and their transposes $\langle\bar{B}\rangle,\langle\bar{E}\rangle$, corresponding to Allen's relations "begun by", "ended by" and their inverses "begins", "ends", provided 
that singleton intervals are included in the temporal structure [21]. HS turns out to be highly undecidable under very weak assumptions on the class of linear orders over which its formulas are interpreted [10]. In particular, undecidability holds for any class of linear orders that contains at least one linear order with an infinitely ascending or descending sequence, thus including the natural time flows $\mathbb{N}, \mathbb{Z}, \mathbb{Q}$, and $\mathbb{R}$. In fact, undecidability occurs even without infinitely ascending/descending sequences: undecidability also holds for any class of linear orders with unboundedly ascending sequences, that is, for any class such that for every $n$, there is a structure in the class with an ascending sequence of length at least $n$, e.g., for the class of all finite linear orders. In [12], Lodaya sharpens the undecidability of HS showing that the two modalities $\langle B\rangle,\langle E\rangle$ suffice for undecidability over dense linear orders (in fact, the result applies to the class of all linear orders [9]).

The recent identification of expressive decidable fragments of HS, whose decidability does not depend on simplifying semantic assumptions such as locality and homogeneity [9], shows that such a trade-off between expressiveness and decidability of interval temporal logics can actually be overcome. The most significant ones are the logic $B \bar{B}$ (resp., $E \bar{E}$ ) of Allen's "begun by/begins" (resp., "ended by/ends") relations [9], the logic $A \bar{A}$ of temporal neighborhood, whose modalities correspond to Allen's "meets/met by" relations (it can be easily shown that Allen's "before/after" relations can be expressed in $A \bar{A}$ ) [8], and the logic $\mathrm{D} \overline{\mathrm{D}}$ of the subinterval/superinterval relations, whose modalities correspond to Allen's "contains/during" relations [14]. In this paper, we focus our attention on the logic $A \bar{A} B \bar{B}$ that joins $B \bar{B}$ and $A \bar{A}$ (the case of $A \bar{A} E \bar{E}$ is fully symmetric). The decidability of $B \bar{B}$ (resp., $E \bar{E}$ ) can be proved by translating it into the point-based propositional temporal logic of linear time with temporal modalities $\mathrm{F}$ (sometime in the future) and $\mathrm{P}$ (sometime in the past), which has the finite (pseudo-)model property and is decidable [9]. Unfortunately, such a reduction to point-based temporal logics does not work for most interval temporal logics as their propositional variables are evaluated over pairs of points and translate into binary relations. This is the case with $A \bar{A}$. Unlike the case of $B \bar{B}$ (resp., $E \bar{E}$ ), when dealing with $A \bar{A}$ one cannot abstract away from the left (resp., right) endpoint of intervals, as contradictory formulas may hold over intervals with the same right (resp., left) endpoint and a different left (resp., right) one. The decidability of $A \bar{A}$, over various classes of linear orders, has been proved by Bresolin et al. [3] by reducing its satisfiability problem to that of the two-variable fragment of first-order logic over the same classes of linear orders [17]. An optimal (NEXPTIME) tableau-based decision procedure for $A \bar{A}$ over the integers has been given in [5] and later extended to the classes of all (resp., dense, discrete) linear orders [6], while a decidable metric extension of the future fragment of $A \bar{A}$ over the natural numbers has been proposed in [7] and later extended to the full logic [4]. Finally, a number of undecidable extensions of $A \bar{A}$ have been given in $[2,3]$.

In [16], Montanari et al. consider the effects of adding the modality $\langle A\rangle$ to $B \bar{B}$, interpreted over the natural numbers. They show that $A B \bar{B}$ retains the 
simplicity of its constituents, but it improves a lot on their expressive power. In particular, besides making it possible to easily encode the until operator of point-based temporal logic (this is possible neither with $B \bar{B}$ nor with $A$ ), $A B \bar{B}$ allows one to express accomplishment conditions as well as metric constraints. Such an increase in expressiveness is achieved at the cost of an increase in complexity: the satisfiability problem for $A B \bar{B}$ is EXPSPACE-complete (that for $A$ is NEXPTIME-complete). In this paper, we show that the addition of the modality $\langle\bar{A}\rangle$ to $A B \bar{B}$ drastically changes the characteristics of the logic. First, decidability is preserved (only) if $A \bar{A} B \bar{B}$ is interpreted over finite linear orders, but the satisfiability problem is not primitive recursive anymore. Moreover, we show that the addition of any modality in the set $\{\langle\mathrm{D}\rangle,\langle\overline{\mathrm{D}}\rangle,\langle\mathrm{E}\rangle,\langle\overline{\mathrm{E}}\rangle,\langle\mathrm{O}\rangle,\langle\overline{\mathrm{O}}\rangle\}$ (modalities $\langle\mathrm{O}\rangle,\langle\overline{\mathrm{O}}\rangle$ correspond to Allen's "overlaps/overlapped by" relations) to $A \bar{A} B \bar{B}$ leads to undecidability. This allows us to conclude that $A \bar{A} B \bar{B}$, interpreted over finite linear orders, is maximal with respect to decidability. Next, we prove that the satisfiability problem for $A \bar{A} B \bar{B}$ becomes undecidable when it is interpreted over any class of linear orders that contains at least one linear order with an infinitely ascending sequence, thus including the natural time flows $\mathbb{N}, \mathbb{Z}, \mathbb{Q}$, and $\mathbb{R}$. As matter of fact, we prove that the addition of $B$ to $A \bar{A}$ suffices to yield undecidability (the proof can be easily adapted to the case of $\bar{B})$. Paired with undecidability results in $[2,3]$, this shows the maximality of $A \bar{A}$ with respect to decidability when interpreted over these classes of linear orders.

\section{The interval temporal logic $A \bar{A} B \bar{B}$}

In this section, we first give syntax and semantics of the logic $A \bar{A} B \bar{B}$. Then, we introduce the basic notions of atom, type, and dependency. We conclude the section by providing an alternative interpretation of $A \bar{A} B \bar{B}$ over labeled grid-like structures (such an interpretation is quite common in the interval temporal logic setting).

\subsection{Syntax and semantics}

Given a set Prop of propositional variables, formulas of $A \bar{A} B \bar{B}$ are built up from Prop using the boolean connectives $\neg$ and $\vee$ and the unary modal operators $\langle A\rangle,\langle\bar{A}\rangle,\langle B\rangle,\langle\bar{B}\rangle$. As usual, we shall take advantage of shorthands like $\varphi_{1} \wedge \varphi_{2}=$ $\neg\left(\neg \varphi_{1} \vee \neg \varphi_{2}\right),[A] \varphi=\neg\langle A\rangle \neg \varphi,[B] \varphi=\neg\langle B\rangle \neg \varphi, T=p \vee \neg p$, and $\perp=p \wedge \neg p$, with $p \in \mathcal{P}$ rop. Hereafter, we denote by $|\varphi|$ the size of $\varphi$.

We interpret formulas of $A \bar{A} B \bar{B}$ in interval temporal structures over finite linear orders with the relations "meets", "met by", "begins", and "begun by". Precisely, given $N \in \mathbb{N}$, we define $\mathbb{I}_{N}$ as the set of all (non-singleton) closed intervals $[x, y]$, with $0 \leq x<y \leq N$. For any pair of intervals $[x, y],\left[x^{\prime}, y^{\prime}\right] \in \mathbb{I}_{N}$, Allen's relations "meets" $A$, "met by" $\bar{A}$, "begun by" $B$, and "begins" $\bar{B}$ are defined as follows:

- "meets": $[x, y] A\left[x^{\prime}, y^{\prime}\right]$ iff $y=x^{\prime}$;

- "met by": $[x, y] \bar{A}\left[x^{\prime}, y^{\prime}\right]$ iff $x=y^{\prime}$; 
- "begun by": $[x, y] B\left[x^{\prime}, y^{\prime}\right]$ iff $x=x^{\prime}$ and $y^{\prime}<y$;

- "begins": $[x, y] \bar{B}\left[x^{\prime}, y^{\prime}\right]$ iff $x=x^{\prime}$ and $y<y^{\prime}$.

Given an interval structure $\mathcal{S}=\left(\mathbb{I}_{\mathrm{N}}, \mathrm{A}, \overline{\mathrm{A}}, \mathrm{B}, \overline{\mathrm{B}}, \sigma\right)$, where $\sigma: \mathbb{I}_{\mathrm{N}} \rightarrow \mathscr{P}(\mathcal{P}$ rop $)$ is a labeling function that maps intervals in $\mathbb{I}_{\mathrm{N}}$ to sets of propositional variables, and an initial interval I, we define the semantics of an $A \bar{A} B \bar{B}$ formula as follows:

- $\mathcal{S}, \mathrm{I} \vDash \mathrm{a}$ iff $\mathrm{a} \in \sigma(\mathrm{I})$, for any $\mathrm{a} \in \mathcal{P}$ rop;

- $\mathcal{S}, \mathrm{I} \vDash \neg \varphi$ iff $\mathcal{S}, \mathrm{I} \neq \varphi$;

- $\mathcal{S}, \mathrm{I} \vDash \varphi_{1} \vee \varphi_{2}$ iff $\mathcal{S}, \mathrm{I} \vDash \varphi_{1}$ or $\mathcal{S}, \mathrm{I} \vDash \varphi_{2} ;$

- for every relation $R \in\{A, \bar{A}, B, \bar{B}\}, \mathcal{S}, I \vDash\langle R\rangle \varphi$ iff there is an interval $J \in \mathbb{I}_{N}$ such that I R J and $\mathcal{S}, J \vDash \varphi$.

Given an interval structure $\mathcal{S}$ and a formula $\varphi$, we say that $\mathcal{S}$ satisfies $\varphi$ if there is an interval $\mathrm{I}$ in $\mathcal{S}$ such that $\mathcal{S}, \mathrm{I} \vDash \varphi$. We say that $\varphi$ is satisfiable if there exists an interval structure that satisfies it. We define the satisfiability problem for $A \bar{A} B \bar{B}$ as the problem of establishing whether a given $A \bar{A} B \bar{B}$-formula $\varphi$ is satisfiable.

\subsection{Atoms, types, and dependencies}

Let $\mathcal{S}=\left(\mathbb{I}_{N}, A, \bar{A}, B, \bar{B}, \sigma\right)$ be an interval structure and $\varphi$ be a formula of $A \bar{A} B \bar{B}$. In the sequel, we shall compare intervals in $\mathcal{S}$ with respect to the set of subformulas of $\varphi$ they satisfy. To do that, we introduce the key notions of $\varphi$-atom and $\varphi$-type.

First of all, we define the closure $\operatorname{Cl}(\varphi)$ of $\varphi$ as the set of all subformulas of $\varphi$ and of their negations (we identify $\neg \neg \alpha$ with $\alpha, \neg\langle A\rangle \alpha$ with $[A] \neg \alpha$, etc.). For technical reasons, we also introduce the extended closure $\mathrm{Cl}^{+}(\varphi)$, which is defined as the set of all formulas in $\mathcal{C l}(\varphi)$ plus all formulas of the forms $\langle\mathrm{R}\rangle \alpha$ and $\neg\langle R\rangle \alpha$, with $R \in\{A, \bar{A}, B, \bar{B}\}$ and $\alpha \in \mathcal{C} l(\varphi)$.

A $\varphi$-atom is any non-empty set $\mathrm{F} \subseteq \mathrm{Cl}^{+}(\varphi)$ such that (i) for every $\alpha \in \mathrm{Cl}^{+}(\varphi)$, we have $\alpha \in \mathrm{F}$ iff $\neg \alpha \notin \mathrm{F}$ and (ii) for every $\gamma=\alpha \vee \beta \in \mathcal{C l}^{+}(\varphi)$, we have $\gamma \in \mathrm{F}$ iff $\alpha \in F$ or $\beta \in F$ (intuitively, a $\varphi$-atom is a maximal locally consistent set of formulas chosen from $\left.\mathrm{Cl}^{+}(\varphi)\right)$. Note that the cardinalities of both sets $\mathcal{C l}(\varphi)$ and $\mathrm{Cl}^{+}(\varphi)$ are linear in the number $|\varphi|$ of subformulas of $\varphi$, while the number of $\varphi$-atoms is at most exponential in $|\varphi|$ (precisely, we have $|\mathcal{C l}(\varphi)|=2|\varphi|,\left|\mathcal{C l}^{+}(\varphi)\right|=18|\varphi|$, and there are at most $2^{9|\varphi|}$ distinct atoms).

We also associate with each interval $\mathrm{I} \in \mathcal{S}$ the set of all formulas $\alpha \in \mathrm{Cl}^{+}(\varphi)$ such that $\mathcal{S}, I \vDash \alpha$. Such a set is called $\varphi$-type of $I$ and it is denoted by $\mathcal{T}_{y p e}(\mathrm{I})$. We have that every $\varphi$-type is a $\varphi$-atom, but not vice versa. Hereafter, we shall omit the argument $\varphi$, thus calling a $\varphi$-atom (resp., a $\varphi$-type) simply an atom (resp., a type).

Given an atom F, we denote by $\mathcal{O} b s(\mathrm{~F})$ the set of all observables of $\mathrm{F}$, namely, the formulas $\alpha \in \mathcal{C l}(\varphi)$ such that $\alpha \in F$. Similarly, given an atom $F$ and a relation $R \in\{A, \bar{A}, B, \bar{B}\}$, we denote by $\mathcal{R e q}_{R}(\mathrm{~F})$ the set of all R-requests of $F$, namely, the formulas $\alpha \in \mathcal{C l}(\varphi)$ such that $\langle R\rangle \alpha \in F$. Note that, for every pair of intervals $\mathrm{I}=(x, y)$ and $J=\left(x^{\prime}, y^{\prime}\right)$ in $\mathcal{S}$, if $y=y^{\prime}$ (resp., $x=x^{\prime}$ ) holds, then 


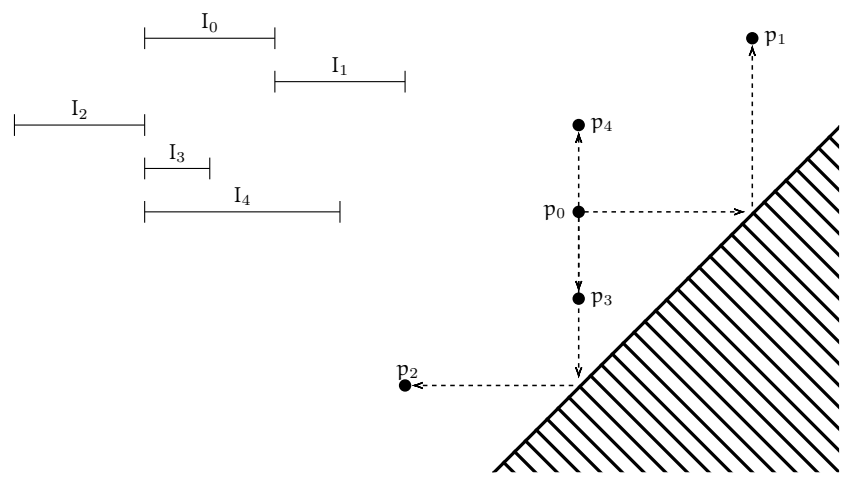

Fig. 1. A compass structure.

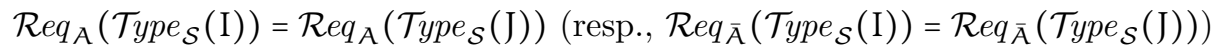
follows. Taking advantage of the above sets, we can define the following relations between atoms $\mathrm{F}$ and $\mathrm{G}$ :

$$
\begin{aligned}
\mathrm{F} \stackrel{\mathrm{A}}{\longrightarrow} \mathrm{G} \quad \text { iff } \quad\left\{\begin{array}{l}
\operatorname{Re}_{\mathrm{A}}(\mathrm{F})=\mathcal{O} b s(\mathrm{G}) \cup \mathcal{R} e q_{\overline{\mathrm{B}}}(\mathrm{G}) \\
\operatorname{Re}_{\mathrm{B}}(\mathrm{G})=\varnothing \\
\mathcal{O} b s(\mathrm{~F}) \subseteq \mathcal{R} e q_{\overline{\mathcal{A}}}(\mathrm{G})
\end{array}\right. \\
\mathrm{F} \stackrel{\mathrm{B}}{\longmapsto} \mathrm{G} \quad \text { iff } \quad\left\{\begin{array}{l}
\mathcal{R} e q_{\mathrm{B}}(\mathrm{F})=\mathcal{O} b s(\mathrm{G}) \cup \mathcal{R} e q_{\mathrm{B}}(\mathrm{G}) \\
\mathcal{R} e q_{\overline{\mathrm{B}}}(\mathrm{G})=\mathcal{O} b s(\mathrm{~F}) \cup \mathcal{R} e q_{\overline{\mathrm{B}}}(\mathrm{F}) .
\end{array}\right.
\end{aligned}
$$

Note that the above relations satisfy a view-to-type dependency, namely, for every pair of intervals $I=[x, y]$ and $I^{\prime}=\left[x^{\prime}, y^{\prime}\right]$, we have

$$
\begin{array}{lll}
x^{\prime}=y \wedge y^{\prime}=y+1 & \text { implies } & \text { Type }_{\mathcal{S}}(I) \stackrel{\wedge}{\longmapsto} \text { Type }_{\mathcal{S}}\left(I^{\prime}\right) \\
x^{\prime}=x \wedge y^{\prime}=y-1 & \text { implies } & \mathcal{T}_{\text {ype }}(I) \stackrel{B}{\longmapsto} \mathcal{T}_{\text {Type }}\left(I_{\mathcal{S}}\left(I^{\prime}\right) .\right.
\end{array}
$$

\subsection{Compass structures}

The logic $A \bar{A} B \bar{B}$ can be equivalently interpreted over the so-called compass structures [20], namely, over grid-like structures. Such an alternative interpretation exploits the existence of a natural bijection between the intervals $I=[x, y]$ and the points $p=(x, y)$ of an $N \times N$ grid such that $x<y$. As an example, Figure 1 depicts five intervals $\mathrm{I}_{0}, \ldots, \mathrm{I}_{4}$ such that $\mathrm{I}_{0} A \mathrm{I}_{1}, \mathrm{I}_{0} \overline{\mathrm{A}} \mathrm{I}_{2}, \mathrm{I}_{0} B \mathrm{I}_{3}$, and $\mathrm{I}_{0} \overline{\mathrm{B}} \mathrm{I}_{4}$, together with the corresponding points $p_{0}, \ldots, p_{4}$ of a discrete grid (note that the four Allen's relations $A, \bar{A}, B, \bar{B}$ between intervals are mapped to corresponding spatial relations between points; for the sake of readability, we name the latter ones as the former ones).

Definition 1. Given an $A \bar{A} B \bar{B}$ formula $\varphi$, a (finite, consistent, and fulfilling) compass ( $\varphi$-)structure of length $\mathrm{N} \in \mathbb{N}$ is a pair $\mathcal{G}=\left(\mathbb{P}_{\mathrm{N}}, \mathcal{L}\right)$, where $\mathbb{P}_{\mathrm{N}}$ is the 
set of points $\mathrm{p}=(\mathrm{x}, \mathrm{y})$, with $0 \leq \mathrm{x}<\mathrm{y} \leq \mathrm{N}$, and $\mathcal{L}$ is function that maps any point $\mathrm{p} \in \mathbb{P}_{\mathrm{N}}$ to a $(\varphi-)$ atom $\mathcal{L}(\mathrm{p})$ in such a way that

- for every relation $\mathrm{R} \in\{\mathrm{A}, \overline{\mathrm{A}}, \mathrm{B}, \overline{\mathrm{B}}\}$ and every pair of points $\mathrm{p}, \mathrm{q} \in \mathbb{P}_{\mathrm{N}}$ such that $\mathrm{p} \mathrm{R} \mathrm{q}$, we have $\mathcal{O} b s(\mathcal{L}(\mathrm{q})) \subseteq \mathcal{R} e q_{\mathrm{R}}(\mathcal{L}(\mathrm{p}))$ (consistency);

- for every relation $R \in\{A, \bar{A}, B, \bar{B}\}$, every point $p \in \mathbb{P}_{N}$, and every formula $\alpha \in \mathcal{R} e q_{\mathrm{R}}(\mathcal{L}(\mathrm{p}))$, there is a point $\mathrm{q} \in \mathbb{P}_{\mathrm{N}}$ such that $\mathrm{p} \mathrm{R} \mathrm{q}$ and $\alpha \in \mathcal{O} b s(\mathcal{L}(\mathrm{q}))$ (fulfillment).

It is easy to see that the (finite, consistent, and fulfilling) compass structures are exactly those structures $\mathcal{G}=\left(\mathbb{P}_{\mathrm{N}}, \mathcal{L}\right)$, with $\mathrm{N} \in \mathbb{N}$, that satisfy the following conditions for all pair of points $p, q$ in $\mathcal{G}$ :

i) if $p=(x, y)$ and $q=(y, y+1)$, then $\mathcal{L}(p) \stackrel{A}{\longmapsto} \mathcal{L}(q)$;

ii) if $p=(x, y)$ and $q=(x, y+1)$, then $\mathcal{L}(q) \stackrel{B}{\longmapsto} \mathcal{L}(p)$;

iii) if $\mathrm{p}=(\mathrm{y}-1, \mathrm{y})$, then $\mathcal{R e q}_{\overline{\mathrm{A}}}(\mathcal{L}(\mathrm{p}))=\bigcup_{0 \leq x<y-1} \mathcal{O} b s(\mathcal{L}(\mathrm{x}, \mathrm{y}-1))$;

iv) if $\mathrm{p}=(\mathrm{x}, \mathrm{N})$, then $\mathcal{R e}_{\mathrm{A}}(\mathcal{L}(\mathrm{p}))=\varnothing$ and $\mathcal{R} e q_{\overline{\mathrm{B}}}(\mathcal{L}(\mathrm{p}))=\varnothing$.

We say that a compass structure $\mathcal{G}=\left(\mathbb{P}_{\mathrm{N}}, \mathcal{L}\right)$ features a formula $\alpha$ if there is a point $p \in \mathbb{P}_{\mathrm{N}}$ such that $\alpha \in \mathcal{L}(\mathrm{p})$. We conclude the section with the following basic result (the proof is straightforward and thus omitted).

Proposition 1. An $\mathrm{A} \overline{\mathrm{A}} \mathrm{B} \overline{\mathrm{B}}$-formula $\varphi$ is satisfied by some finite interval structure iff it is featured by some finite $\varphi$-compass structure.

\section{Decidability and complexity of the satisfiability problem for $A \bar{A} B \bar{B}$ over finite linear orders}

In this section, we prove that the satisfiability problem for $A \bar{A} B \bar{B}$ interpreted over finite linear orders is decidable, but not primitive recursive. In order to do that, we use a technique similar to [16], namely, we fix a formula $\varphi$ and a finite compass structure $\mathcal{G}=\left(\mathbb{P}_{\mathrm{N}}, \mathcal{L}\right)$ satisfying $\varphi$ and we show that, under suitable conditions, $\mathcal{G}$ can be reduced in length while preserving the existence of atoms featuring $\varphi$. For the sake of brevity, we call contraction the operation that reduces the length of a given compass structure $\mathcal{G}$ while preserving the existence of atoms featuring $\varphi$. Such an operation has been introduced in its simple variant in [16] and it precisely consists of removing the portion of the compass structure $\mathcal{G}$ included between two distinguished rows $y_{0}$ and $y_{1}$ and selecting a subset of atoms from the upper row $y_{1}$ that match with the atoms of the lower row $y_{0}$. Hereafter, we refer the reader to Figure 2 for an intuitive account of the contraction operation (the colored nodes represent the atoms associated with the points of $\mathcal{G}$ ). According to the definition given in [16], the contraction operation is applicable whenever the set of atoms of the lower row $y_{0}$ is included in the set of atoms of the upper row $y_{1}$ (the arrows in Figure 2 represent a matching function $f$ between the atoms of the lower row $y_{0}$ and the atoms of the upper row $y_{1}$ ). Such a condition on the set of atoms associated with the rows $y_{0}$ and $y_{1}$ guarantees the correctness of the contraction operation with 


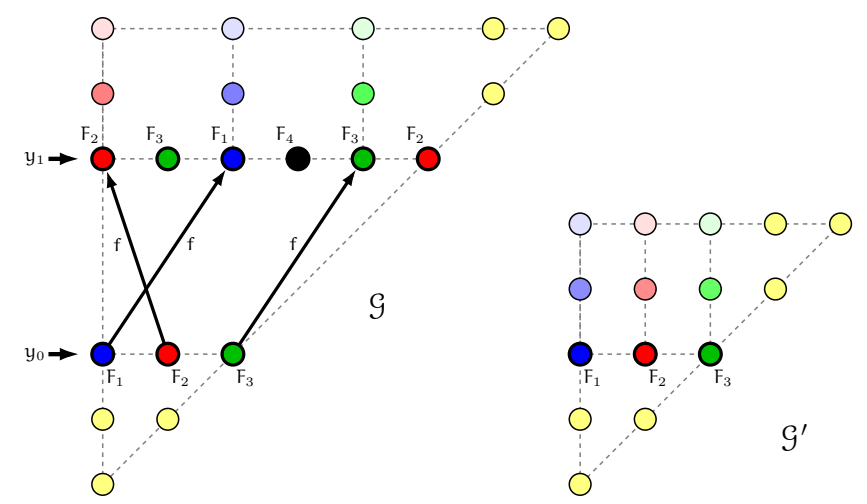

Fig. 2. Contraction of a compass structure.

respect to the definition of consistent and fulfilling compass structure, provided that the use of the modal operator $\langle\bar{A}\rangle$ is avoided. However, in the presence of the modal operator $\langle\bar{A}\rangle$, things get more involved, since some points $p=\left(x, y_{1}\right)$ from the upper row $y_{1}$ (e.g., the one labeled by $F_{4}$ in Figure 2) might be necessary in order to fulfill the $\bar{A}$-requests enforced by other points $p^{\prime}=\left(x^{\prime}, y^{\prime}\right)$, with $x^{\prime}=y_{1}$ and $y^{\prime}>y_{1}$. In the following, we describe a suitable variant of the contraction operation which is applicable to models of $A \bar{A} B \bar{B}$ formulas.

Let us fix an $A \bar{A} B \bar{B}$ formula $\varphi$ that is featured by a finite compass structure $\mathcal{G}=\left(\mathbb{P}_{\mathrm{N}}, \mathcal{L}\right)$. Without loss of generality, we can assume that $\varphi$ is of the form $(\phi \wedge[\mathrm{B}] \perp) \vee(\langle\overline{\mathrm{B}}\rangle \phi) \vee(\langle\overline{\mathrm{B}}\rangle\langle\mathrm{A}\rangle \phi)$ and, furthermore, it belongs to the atom associated with the point $p=(0,1)$ at the bottom of the structure $\mathcal{G}$. Before turning to our main result, we need to introduce some preliminary notation and terminology. For every $1 \leq \mathrm{y} \leq \mathrm{N}$, we denote by $\mathcal{R}_{o} w_{\mathcal{G}}(\mathrm{y})$ the row $\mathrm{y}$ of $\mathcal{G}$, namely, the set of all points $p=(x, y)$ of $\mathcal{G}$. We associate with each row $y$ the set $\operatorname{Shading}_{\mathcal{G}}(\mathrm{y})=\mathcal{L}\left(\mathcal{R}_{\mathrm{O}} w_{\mathcal{G}}(\mathrm{y})\right)$, which consists of the atoms associated with the points in $\mathcal{R}_{\mathcal{G}}(\mathrm{y})$. Clearly, for every pair of atoms $\mathrm{F}, \mathrm{G}$ in $\operatorname{Shading}_{\mathcal{G}}(\mathrm{y})$, we have $\mathcal{R} e q_{\mathrm{A}}(\mathrm{F})=\mathcal{R}_{e} q_{\mathrm{A}}(\mathrm{G})$. We also associate with the row $y$ the function Count $_{\mathcal{G}}(\mathrm{y})$, which maps an atom $\mathrm{F}$ to the number $\mathcal{C o u n t}_{\mathcal{G}}(\mathrm{y})(\mathrm{F})$ of F-labeled points in $\mathcal{R o w}_{\mathcal{G}}(\mathrm{y})$.

In order to deal with $\bar{A}$-requests, we need to introduce the notion of cover of a compass structure. Intuitively, this is a selection of points that fulfills all $\bar{A}$-requests coming from other points (hence the points in a cover should not disappear during the operation of contraction). Formally, a cover of a compass structure $\mathcal{G}=\left(\mathbb{P}_{\mathrm{N}}, \mathcal{L}\right)$ is a subset $\mathrm{C}$ of $\mathbb{P}_{\mathrm{N}}$ that satisfies the following two conditions:

- if $(x, y) \in C$ and $x<y-1$, then $(x, y-1) \in C$ as well;

- for every point $q=(y-1, y) \in \mathbb{P}_{N}$, the set $\mathcal{R} e q_{\bar{A}}(\mathcal{L}(q))$ coincides with the union of the sets $\mathcal{O} b s(\mathcal{L}(p))$ for all $p=(x, y-1) \in C$.

Given a cover $\mathrm{C}$ of $\mathcal{G}$, we extend the notations $\mathcal{R}_{\mathcal{G}}(\mathrm{y}), \operatorname{Shading}_{\mathcal{G}}(\mathrm{y})$, and

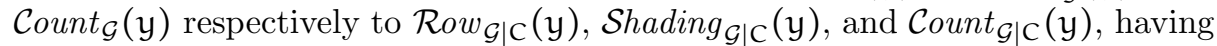
the obvious meaning (e.g., $\mathcal{R}_{o} w_{\mathcal{G} \mid \mathrm{C}}(\mathrm{y})$ is the set of all points of $\mathcal{G}$ along the row 
$\mathrm{y}$ that also belong to $\mathrm{C}$ ). Moreover, we say that a cover is minimal if it does not include properly any other cover. We can easily verify that every minimal cover $\mathrm{C}$ of $\mathcal{G}=\left(\mathbb{P}_{\mathrm{N}}, \mathcal{L}\right)$ satisfies

$$
\begin{aligned}
& \operatorname{Row}_{\mathcal{G} \mid \mathrm{C}}(\mathrm{N})=\varnothing \\
& \left|\mathcal{R}_{\mathcal{G} \mid \mathrm{C}}(\mathrm{y})\right|-1 \leq\left|\mathcal{R}_{\operatorname{lo}} w_{\mathcal{G} \mid \mathrm{C}}(\mathrm{y}-1)\right| \leq\left|\mathcal{R}_{0} w_{\mathcal{G} \mid \mathrm{C}}(\mathrm{y})\right|+|\varphi| .
\end{aligned}
$$

The following proposition shows that, under suitable conditions, a given compass structure $\mathcal{G}$ can be reduced in length while preserving the existence of atoms featuring $\varphi$. Note that such a result can be thought of as a strenghtening of the original "contraction lemma" for structures over the signature $A, B, \bar{B}$ (indeed, if the logic does not allow the modal operator $\langle\bar{A}\rangle$, then the empty set is the unique minimal cover of any compass structure $\mathcal{G}$ and hence the proposition below becomes equivalent to Lemma 3.2 in [16]). For the sake of brevity, hereafter we use $\leq$ to denote the componentwise partial order between functions that map atoms to natural numbers, i.e., $f \leq g$ iff $f(F) \leq g(F)$ holds for all atoms $F$.

Proposition 2. Let $\mathcal{G}=\left(\mathbb{P}_{\mathrm{N}}, \mathcal{L}\right)$ be a compass structure that features a formula $\varphi$ in its bottom row. If there exist a cover $\mathrm{C}$ of $\mathcal{G}$ and two rows $\mathrm{y}_{0}$ and $\mathrm{y}_{1}$ in $\mathcal{G}$, with $1<\mathrm{y}_{0}<\mathrm{y}_{1} \leq \mathrm{N}$, such that

i) Shading $_{\mathcal{G}}\left(\mathrm{y}_{0}\right) \subseteq$ Shading $_{\mathcal{G}}\left(\mathrm{y}_{1}\right)$,

ii) $\operatorname{Count}_{\mathcal{G}}\left(\mathrm{y}_{0}\right) \geq$ Count $_{\mathcal{G} \mid \mathrm{C}}\left(\mathrm{y}_{1}\right)$,

then there exists a compass structure $\mathcal{G}^{\prime}$ of length $\mathrm{N}^{\prime}<\mathrm{N}$ that features $\varphi$.

On the grounds of Proposition 2, it makes sense to restrict ourselves to the minimal models of $\varphi$ and, in particular, to those compass structures $\mathcal{G}=\left(\mathbb{P}_{\mathrm{N}}, \mathcal{L}\right)$ that feature $\varphi(=(\phi \wedge[\mathrm{B}] \perp) \vee(\langle\overline{\mathrm{B}}\rangle \phi) \vee(\langle\overline{\mathrm{B}}\rangle\langle\mathrm{A}\rangle \phi))$ in the bottom row and that cannot be contracted. The above argument leads to a non-deterministic procedure that decides whether a given formula $\phi$ is satisfied by a (contractionfree) interval structure $\mathcal{S}$. The pseudo-code of such an algorithm is given in Figure 3: the variable $\Delta$ represents the value $\mathrm{N}-\mathrm{y}+1$, where $\mathrm{N}$ is the length of the model $\mathcal{G}$ to be guessed and $\mathrm{y}$ is the current row (note that we cannot use $\mathrm{y}$ in place of $\Delta$ since there is no a priori bound on the length $\mathrm{N}$ of the model), the variable $F_{\Delta}$ represents the atom associated with the rightmost point $p=(y-1, y)$ along the current row $y$, the variable $S_{\Delta}$ represents an over-approximation of the set $\mathcal{S h a d i n g}_{\mathcal{G}}(\mathrm{y})$, and the variable $\mathrm{C}_{\Delta}$ represents the function $\mathcal{C}_{\text {ount }} \mathrm{G}_{\mathcal{G} \mid \mathrm{C}}(\mathrm{y})$ for a suitable cover $\mathrm{C}$ of $\mathcal{G}$ (note that the content of such a variable can be guessed because the sum of its values is bounded in virtue of Equation 1).

The decidability of the satisfiability problem for $A \bar{A} B \bar{B}$ interpreted over finite linear orders is thus reduced to a proof of termination, soundness, and completeness for the algorithm given in Figure 3 as formally stated by Theorem 1 (its proof is reported in [15]). As a matter of fact, termination relies on the following crucial lemma, which is often attributed to Dickson.

Lemma 1 (Dickson's Lemma). Let $\left(\mathbb{N}^{k}, \leq\right)$ be the $k$-dimensional vector space over $\mathbb{N}$ equipped with the componentwise partial order $\leq$. Then, $\left(\mathbb{N}^{k}, \leq\right)$ admits 
that suitable disequalities of the form $c(t+1) \leq c(t)+h$, with $h \in\{-1,0,1\}$, hold between the values of the counter $c$ at consecutive time instants. This can be done by enforcing the existence of a surjective partial function $\mathrm{g}$ from the set of $c$-labeled unit-length intervals corresponding to the time instant $t$ to the set of $c$ labeled unit-length intervals corresponding to the next time instant $t+1$. Finally, we exploit the fact that surjective partial functions between sets of unit-length intervals can be specified in the logic $A \bar{A} B$.

\section{Undecidabiliy is the rule, decidability the exception}

We conclude the paper by proving that $A \bar{A} B \bar{B}$, interpreted over finite linear orders, is maximal with respect to decidability. The addition of a modality for any one of the remaining Allen's relations, that is, of any modality in the set $\{\langle\mathrm{D}\rangle,\langle\overline{\mathrm{D}}\rangle,\langle\mathrm{E}\rangle,\langle\overline{\mathrm{E}}\rangle,\langle\mathrm{O}\rangle,\langle\overline{\mathrm{O}}\rangle\}$, indeed leads to undecidability. The proof of the following theorem can be found in [15].

Theorem 3. The satisfiability problem for the logic $\mathrm{A} \overline{\mathrm{A}} \mathrm{B} \overline{\mathrm{B}} \mathrm{D}$ (resp., $\mathrm{A} \overline{\mathrm{A}} \mathrm{B} \overline{\mathrm{B}} \overline{\mathrm{D}}$, $\mathrm{A} \overline{\mathrm{A}} \mathrm{B} \overline{\mathrm{B}} \mathrm{E}, \mathrm{A} \overline{\mathrm{A}} \mathrm{B} \overline{\mathrm{B}} \overline{\mathrm{E}}, \mathrm{A} \overline{\mathrm{A}} \mathrm{B} \overline{\mathrm{B} O}, \mathrm{~A} \overline{\mathrm{A}} \mathrm{B} \overline{\mathrm{B}} \overline{\mathrm{O}})$, interpreted over finite linear orders, is undecidable.

It is possible to show that the satisfiability problem for $A \bar{A} B \bar{B}$ (in fact, this holds for its proper fragment $A \bar{A} B$ ) becomes undecidable if we interpret it over any class of linear orders that contains at least one linear order with an infinitely ascending sequence. It follows that, in particular, it is undecidable when $A \bar{A} B \bar{B}$ is interpreted over natural time flows like $\mathbb{N}, \mathbb{Z}, \mathbb{Q}$, and $\mathbb{R}$. We first consider the satisfiability problem for $A \bar{A} B$ interpreted over $\mathbb{N}$. By definition, $\varphi$ is satisfiable over $\mathbb{N}$ if there exists an interval structure of the form $\mathcal{S}=\left(\mathbb{I}_{\omega}, A, \bar{A}, B, \sigma\right)$, with $\mathbb{I}_{\omega}=\{[x, y]: 0 \leq x<y<\omega\}$ and $\sigma: \mathbb{I}_{\omega} \rightarrow \mathscr{P}(\mathcal{P}$ rop $)$, that satisfies it. A straightforward adaptation of the proof of Theorem 2 (see the proof of Theorem 4 in [15]) shows that an undecidable variant of the universal reachability problem for lossy counter machines, called "structural termination" [13], is reducible to the satisfiability problem for $A \bar{A} B$ interpreted over interval structures of the form $\mathcal{S}=\left(\mathbb{I}_{\omega}, A, \overline{\bar{A}}, B, \sigma\right)$. It immediately follows that the latter problem is undecidable as well. Such a negative result can be easily transferred to any class of linear orders that contains at least one linear order with an infinitely ascending sequence.

Theorem 4. The satisfiability problem for the logic $\mathrm{A} \overline{\mathrm{A}} \mathrm{B}$, and hence that for the logic $\mathrm{A} \overline{\mathrm{A}} \mathrm{B} \overline{\mathrm{B}}$, interpreted over over any class of linear orders that contains at least one linear order with an infinitely ascending sequence is undecidable.

\section{Conclusions}

In this paper, we proved that the satisfiability problem for $A \bar{A} B \bar{B}$, interpreted over finite linear orders, is decidable, but not primitive recursive. We also showed that all proper extensions of $A \bar{A} B \bar{B}$ with a modality corresponding to one of the 
remaining Allen's relations yields undecidability, thus proving maximality of $A \bar{A} B \bar{B}$ with respect to finite linear orders. Moreover, we proved that the satisfiability problem for $A \bar{A} B$ (in fact, the proof for $A \bar{A} B$ can be adapted to $A \bar{A} \bar{B}$ ), interpreted over any class of linear orders that contains at least one linear order with an infinitely ascending sequence, is undecidable. The same results hold for $A \bar{A} E$ and $A \bar{A} \bar{E}$, provided that we replace the infinitely ascending sequence by an infinitely descending one. As Bresolin et al. already proved that the extension of $A \bar{A}$ with the operator $\langle\mathrm{D}\rangle$ (resp., $\langle\overline{\mathrm{D}}\rangle,\langle\mathrm{O}\rangle,\langle\overline{\mathrm{O}}\rangle$ ) is undecidable $[2,3]$, maximality of $A \bar{A}$, interpreted over any class of linear orders that contains at least one linear order with an infinitely ascending/descending sequence, immediately follows. As a matter of fact, this is the first case in the interval temporal logic setting where the decidability/undecidability of a logic depends on the class of linear orders in which it is interpreted (a similar result has been proved by Ouaknine and Worrell for point-based metric temporal logics [18]).

\section{Acknowledgments}

We would like to acknowledge the financial support from the PRIN project Innovative and multi-disciplinary approaches for constraint and preference reasoning and the GNCS project Logics, automata, and games for the formal verification of complex systems.

\section{References}

[1] J.F. Allen. Maintaining knowledge about temporal intervals. Communications of the Association for Computing Machinery, 26(11):832-843, 1983.

[2] D. Bresolin, D. Della Monica, V. Goranko, A. Montanari, and G. Sciavicco. Decidable and undecidable fragments of Halpern and Shoham's interval temporal logic: towards a complete classification. In Proc. of the 15th Int. Conference on Logic for Programming, Artificial Intelligence, and Reasoning (LPAR), volume 5330 of LNCS, pages 590-604. Springer, 2008.

[3] D. Bresolin, V. Goranko, A. Montanari, and G. Sciavicco. Propositional interval neighborhood logics: expressiveness, decidability, and undecidable extensions. Annals of Pure and Applied Logic, 161(3):289-304, 2009.

[4] D. Bresolin, D. Della Monica, V. Goranko, A. Montanari, C. Robinson, and G. Sciavicco. Metric Propositional Neighborhood Logics. Research Report UDMI/2010/02, Dept. of Mathematics and Computer Science, University of Udine, 2010. http://users.dimi.uniud.it/ angelo.montanari/rr201002.pdf.

[5] D. Bresolin, A. Montanari, and P. Sala. An optimal tableau-based decision algorithm for Propositional Neighborhood Logic. In Proc. of the 24th Int. Symposium on Theoretical Aspects of Computer Science (STACS), volume 4393 of $L N C S$, pages 549-560. Springer, 2007.

[6] D. Bresolin, A. Montanari, P. Sala, and G. Sciavicco. Tableau-based decision procedures for Propositional Neighborhood Logic. Research Report UDMI/2010/01, Dept. of Mathematics and Computer Science, University of Udine, 2010. http://users.dimi.uniud.it/ angelo.montanari/rr201001.pdf. 
[7] D. Bresolin, V. Goranko A. Montanari, and G. Sciavicco. Right propositional neighborhood logic over natural numbers with integer constraints for interval lengths. In Proc. of the 7th IEEE Int. Conference on Software Engineering and Formal Methods (SEFM), pages 240-249. IEEE Comp. Society Press, 2009.

[8] V. Goranko, A. Montanari, and G. Sciavicco. Propositional interval neighborhood temporal logics. Journal of Universal Computer Science, 9(9):1137-1167, 2003.

[9] V. Goranko, A. Montanari, and G. Sciavicco. A road map of interval temporal logics and duration calculi. Applied Non-classical Logics, 14(1-2):9-54, 2004.

[10] J.Y. Halpern and Y. Shoham. A propositional modal logic of time intervals. Journal of the ACM, 38:279-292, 1991.

[11] H. Kamp and U. Reyle. From Discourse to Logic: Introduction to Modeltheoretic Semantics of Natural Language, Formal Logic and Discourse Representation Theory, Volume 42 of Studies in Linguistics and Philosophy. Springer, 1993.

[12] K. Lodaya. Sharpening the undecidability of interval temporal logic. In Proc. of the 6th Asian Computing Science Conference on Advances in Computing Science, volume 1961 of LNCS, pages 290-298. Springer, 2000.

[13] R. Mayr. Undecidable problems in unreliable computations. Theoretical Computer Science, 297(1-3):337-354, 2003.

[14] A. Montanari, G. Puppis, and P. Sala. A decidable spatial logic with coneshaped cardinal directions. In Proc. of the 18th EACSL Annual Conference on Computer Science Logic (CSL), volume 5771 of LNCS, pages 394-408. Springer, 2009.

[15] A. Montanari, G. Puppis, and P. Sala. Maximal decidable fragments of Halpern and Shoham's modal logic of intervals. Research Report UDMI/2010/04, Dept. of Mathematics and Computer Science, University of Udine, 2010. http://users.dimi.uniud.it/ angelo.montanari/rr201004.pdf.

[16] A. Montanari, G. Puppis, P. Sala, and G. Sciavicco. Decidability of the interval temporal logic $A B \bar{B}$ over the natural numbers. In Proc. of the 27th Int. Symposium on Theoretical Aspects of Computer Science (STACS), pages 597-608, 2010.

[17] M. Otto. Two variable first-order logic over ordered domains. Journal of Symbolic Logic, 66(2):685-702, 2001.

[18] J. Ouaknine and J. Worrell. On the decidability and complexity of metric temporal logic over finite words. Logical Methods in Computer Science, $3(1): 1-27,2007$.

[19] Ph. Schnoebelen. Verifying lossy channel systems has nonprimitive recursive complexity. Information Processing Letters, 83(5):251-261, 2002.

[20] Y. Venema. Two-dimensional modal logics for relation algebras and temporal logic of intervals. ITLI prepublication series LP-89-03, University of Amsterdam, 1989.

[21] Y. Venema. A modal logic for chopping intervals. Journal of Logic and Computation, 1(4):453-476, 1991. 